\title{
Pediatric Nurses Experience with Integrated Vital Signs Device in Triage Room
}

\author{
Jose BARBUDO III ${ }^{\mathrm{a}, 1}$, Rocky SAN PEDRO ${ }^{\mathrm{a}}$ and Rajvir SINGH ${ }^{\mathrm{a}}$

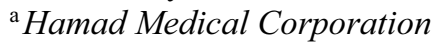

\begin{abstract}
Keywords. Clinical data integration, human factors, human-computer interaction, system implementation
\end{abstract}

\section{Introduction}

Technology has played a defining role in promotion of patient safety and availability of patient information almost instantaneous across the health care continuum. Integration of vital signs monitoring device to the electronic health record (EHR) were expected to communicate patient assessment directly to the clinical information system preventing the use of paper, risk of documentation error, improved patient identification and reduce the delay of data entry of vital signs results which was necessary to be accessed by emergency healthcare providers.

The main aim was to explore what are triage nurses' experiences in using integrated vital signs devices in their interactions with pediatric patients. Specifically, (1) explore and describe the triage nurse's behavior, (2) how nurses cope to overcome the challenges in using the integrated vital signs during assessment of pediatric patients; and (3) to offer insights to other healthcare professionals, researchers and hospital administrator into the use of integrated vital signs device suitable for pediatric patients.

\section{Approach}

The researchers used phenomenological qualitative method to gain a deeper understanding of pediatric nurses' experience with integrated vital signs device in triage room of two pediatric emergency departments of Hamad Medical Corporation, a multihospital system in Qatar. Responses were collected from twenty-five (25) pediatric nurses working in triage room using semi-structured interview. Colaizzi approach was used for content analysis. The data was organized and sorted using computerized qualitative analysis software (nVivo). The data were categorized according to themes to derive meaning significant to the phenomenon of the study.

1 Corresponding Author, Jose Barbudo III, Nursing Informatics Coordinator, Hamad Medical Corporation, Doha Qatar, P.O. 3050; E-mail: jbarbudo@hamad.qa. 


\section{Results}

The study revealed following themes derived were actual use, perceived usefulness, benefits realized, barriers to adoption and coping strategies. Nurses perceived the benefits of the integrated vital signs as a positive element in the triage room and there were opportunities to improve in the current integration workflow pediatric triage setting. Motivating factors of using the integrated vital signs device were identified such as accuracy, ease of use and patient safety while barriers to adoption where ability to modify values, managing patient behavior, issues related to data capture and workflow deviations.

The results of the study highlight the perception of nurses at the benefits of the integration as well as the barriers and the corresponding coping strategies. The coping strategies arise as the end-users faced the challenges/barriers identified in the integration. These elements are considered determinants for the acceptance of the integrative system by the nursing staff and have a direct impact on their actual use.

\section{Conclusions}

Overall, despite of the barriers encountered participants used coping strategies and positively acknowledged that the use of integrated vital signs device can lead to more accurate documentation and save time as part of the triage assessment. The researchers recommend that future research should also encompass the perspectives of physicians and patients on the use of integrated medical devices to improve assessment in triage and prevent prolonging triage time.

\section{Acknowledgement}

The researchers are grateful for the support of the local nursing administration and nursing informatics management in the conduct of the research.

\section{References}

[1] Meccariello, Perkins, Quigley, Rock, Qiu. Vital time savings: Evaluating the use of an automated vital signs documentation system on a medical/surgical unit. Journal of Health Information Management, Fall 2010, Volume 24, Number 4, Pages 46-51.

[2] Skyttberg N, Vicente J, Chen R, Blomqvist H, Koch S. How to improve vital sign data quality for use in clinical decision support systems? A qualitative study in nine Swedish emergency departments. BMC Med Inform Decision Making. 2016 Jun 4; 16:61. doi: 10.1186/s12911-016-0305-4

[3] Wilson SJ, Wong D, Pullinger R, Way R, Clifton D, Tarassenko L (n.d.). Analysis of a data-fusion system for continuous vital sign monitoring in an emergency department. Retrieved August 8, 2018, from https://pdfs.semanticscholar.org/4476/2025eaff0fbc135df86e0093bd116c73491b.pdf

[4] Stevenson JE. (2011). Documentation of vital signs in electronic health records: A patient safety issue (Doctoral dissertation). Retrieved from http://etheses.whiterose.ac.uk/12704/1/PhD\%20Thesis \%20Jean\%20E\%20Stevenson.pdf

[5] Girardo, L et al. (2018). Exploring nurses' perceptions and expectations toward a BCMA implementation using a mobile app and workstations as a change management strategy. Studies in Health Technology and Informatics, 134-138. Retrieved from https://www.ncbi.nlm.nih.gov/pubmed/29857405 